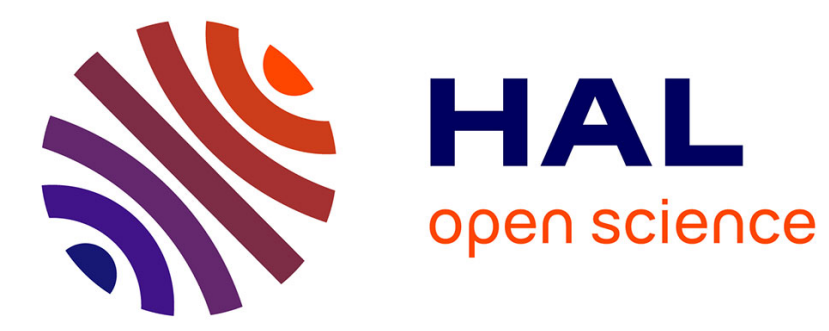

\title{
Propagation of a continuously supplied gravity current head down bottom slopes
}

\author{
A. Martin, M.E. Negretti, M. Ungarish, T. Zemach
}

\section{To cite this version:}

A. Martin, M.E. Negretti, M. Ungarish, T. Zemach. Propagation of a continuously supplied gravity current head down bottom slopes. Physical Review Fluids, 2020, 5 (5), 10.1103/PhysRevFluids.5.054801 . hal-02958134

\section{HAL Id: hal-02958134 \\ https://hal.science/hal-02958134}

Submitted on 23 Nov 2020

HAL is a multi-disciplinary open access archive for the deposit and dissemination of scientific research documents, whether they are published or not. The documents may come from teaching and research institutions in France or abroad, or from public or private research centers.
L'archive ouverte pluridisciplinaire HAL, est destinée au dépôt et à la diffusion de documents scientifiques de niveau recherche, publiés ou non, émanant des établissements d'enseignement et de recherche français ou étrangers, des laboratoires publics ou privés. 


\title{
Propagation of a continuously supplied gravity current head down bottom slopes
}

\author{
A. Martin $\odot^{*}$ and M. E. Negretti \\ LEGI, UGA/CNRS UMR 5519, Grenoble Cedex 9, France \\ M. Ungarish \\ Computer Science Department, Technion Israel Institute of Technology, Haifa 32000, Israel \\ T. Zemach \\ Computer Science Department, Tel-Hai College, Upper Galilee, 1220800 Israel
}

\begin{abstract}
We present a combined theoretical-experimental investigation of the downslope propagation of a gravity current sustained by a source. The current propagates first on a horizontal bottom, then on a downslope. We focus on the case when the current at the ridge (point where donwslope begins) has a stable interface $(\mathrm{Ri}>0.25)$ and is critical with $F=1$, where Ri and $F$ are the bulk Richardson and flow Froude numbers. We derive the equations that govern the nose propagation and speed using a shallow-water (SW) model, in which the nose is a jump matched to characteristics emitted at the ridge. This provides a self-contained prediction for the speed of propagation $u_{N}$ and position $\xi_{N}$ of the nose. The predicted $u_{N}$ increases with time and distance $\xi$ from the ridge. Since Ri decreases with $\xi$ in the tail behind the nose, appearance of instabilities at a certain traveled distance determines the domain of validity of the SW solution. A good agreement is reported with various experiments with different initial conditions at the ridge and slope angles (both fixed and changing with distance from the ridge). It is shown that the nose velocity is always less than the maximum velocity within the current head, which corresponds to the speed of the characteristics released at the ridge that catch on the current head.
\end{abstract}

\section{INTRODUCTION}

There is a long-standing interest in gravity driven flows since they are ubiquitous in natural and man-made environments. Examples include snow avalanches [1], dust or pyrocastic flows [2], and bottom water formation in the oceans [3].

The typical system of gravity current, which has received much attention in experimental, theoretical, and numerical investigations, is of lock-release of a dense fluid over a horizontal bottom in a tank filled with a slightly less dense fluid. Once the barrier is removed, the heavier fluid forms a well-defined frontal region after an initial accelerating transient regime. The Reynolds number is large, and the motion is governed by a buoyancy-inertia balance. After a short accelerating transient regime, the current propagates with a constant velocity $u_{N}$ for a period referred to as the slumping stage; then it enters into a self-similar stage with decaying $u_{N}$ and eventually the viscous forces begin to dominate inertia. These features are well documented by experimental investigations

\footnotetext{
*antoine_martin@orange.fr
} 


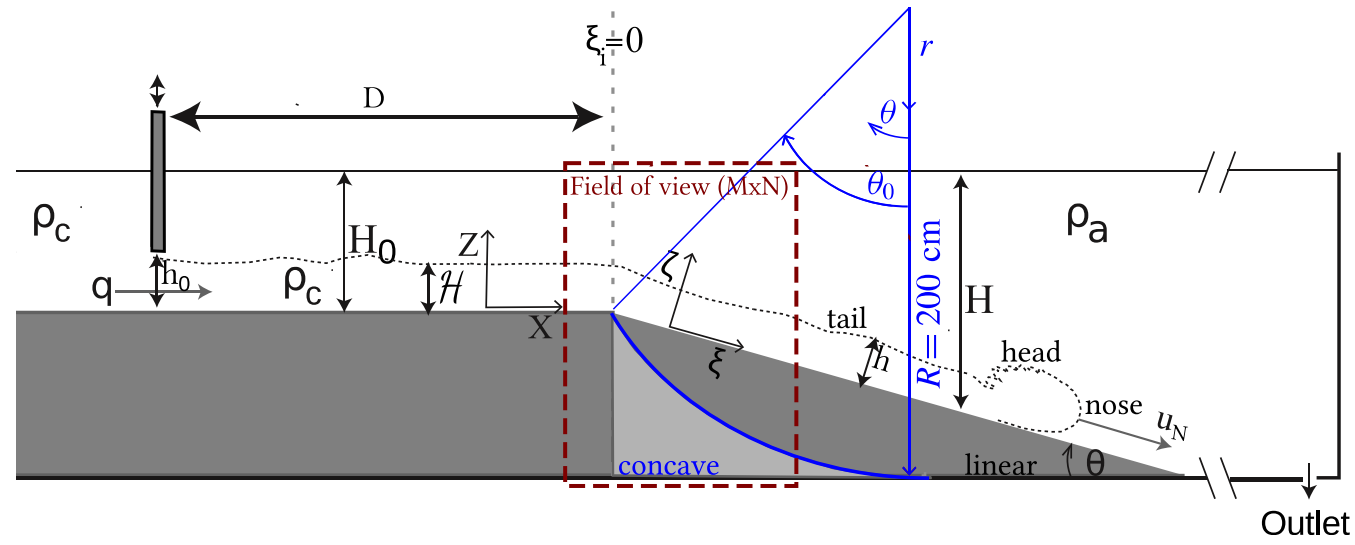

FIG. 1. Sketch of the experimental setup and definition of notations. The horizontal component of the velocity is $\mathcal{U}$ and the along-slope component of the velocity is $u$. All of the notations are listed in Table I.

[e.g., 2,4] and are amenable to interpretation and prediction by theoretical models of the shallowwater (SW) type (see [5] and references herein).

An important property of the horizontal gravity current is the stability of the interface: the bulk Richardson number $\mathrm{Ri}=\left(g^{\prime} h\right) / u^{2}$ is fairly large for a long time and distance of propagation (see [6]). Herein, $g^{\prime}=g \frac{\rho_{c}-\rho_{a}}{\rho_{a}}$ is the reduced gravity, where $\rho_{c}$ and $\rho_{a}$ are respectively the density of the gravity current and the ambient, $h$ is the depth of the current, and $u$ the depth-averaged speed of the current, as shown in Fig. 1. In such a configuration, the shallow water model combined with the characteristics method and a jump condition can be used to predict the nose behavior [7].

Several previous studies considered a continuous supply of the gravity current with the constant flux $B_{0}=g^{\prime} q$, where $q$ is the initial flow rate per unit of width imposed by a source at the top of the slope (Britter and Linden [8] for the head; Ellison and Turner [9] and Pawlak and Armi [10] for the tail). In these studies, the current emitted at the source is in the $\mathrm{Ri}<\mathrm{Ri}_{c} \approx 0.25$ regime at the ridge and Kelvin-Helmholtz vortices; entrainment and drag are present from the beginning. We can expect that the speed in the steady-state tail is, from the beginning, of the order of magnitude $\left(B_{0} / \mathrm{Ri}_{c}\right)^{1 / 3} \approx 1.6 B_{0}^{1 / 3}$ (see Fig. 9 in Ellison and Turner [9]). Britter and Linden [8] derived the semiempirical approximation for the (constant) nose propagation speed $u_{N}=1.5 B_{0}^{1 / 3}$. This result is in fair agreement with experiments (within about $\pm 15 \%$ ) for a wide range of slope angles. The flow is affected by entrainment and drag and, due to the interfacial instabilities, the initial conditions on the characteristics of a SW model are uncertain and need to be verified.

In Negretti et al. [11] and Martin et al. [12] (N17 and M19) the source was positioned at some upstream position (see Fig. 1), producing different initial conditions than those usually used in the literature [e.g., 10,13]. In N17 and M19 the tail of the gravity current starts on the slope without any entrainment due to an initial stable interface of the current whereas. in the previous literature, the tail of the gravity current starts on the slope with a large entrainment due to an initial unstable interface of the current. Negretti et al. [11] demonstrate that beyond the straightforward separation into "formation" and "steady-state" stages, additional subdivisions for the second "steady-state" stage are required. They show that for a gravity flow released far upstream of the edge, the rear part of the gravity flow on the slope has a stable interface, while more downslope the flow displays an increasing speed and decreasing $\mathrm{Ri}$, until a point where the interface becomes unstable. Negretti et al. [11] have shown for such a configuration that the motion of the nose can be well approximated by a "free-fall" behavior: assuming that the head is a rigid body, the potential energy in the reduced-gravity field is converted into kinetic energy as the nose advances from the initial height $Z_{i}$ to $Z_{N}(t)=Z_{i}-\tan \theta\left[X_{N}(t)-X_{i}\right]$, where $(X, Z)$ are the horizontal and vertical coordinates, $\theta$ the 
slope angle, as depicted in Fig. 1, and $t$ denotes time. This consideration yields the formula

$$
u_{N}=\left[2 S_{2} g^{\prime}\left(Z_{i}-Z_{N}\right)+u_{N i}^{2}\right]^{1 / 2},
$$

where $S_{2}$ is a shape factor related to the shape of the velocity and density vertical profiles [14] and can be interpreted as an adjustable constant. The advantage of this approximation is that it reproduces surprisingly well the maximal free stream velocity in the head [11]. The deficiency is that this is actually a curve fit which lacks theoretical justification, needs empirical inputs, and does not provide insight about the mechanism that governs the propagation of the nose.

Here, we consider the complex system of Negretti et al. [11] and Martin et al. [12] in which the current is produced and sustained by a continuous supply of dense fluid (constant flux $B_{0}$ ) and the propagation is over an inclined bottom. In this case, the flow has two main stages: the time-dependent formation of a stream (tail) following the nose moving with $u_{N}$, then a possible steady state along the entire slope or part of it. Intuitively, the high-Reynolds-number stream is expected to accelerate and contract during propagation. This suggests that time dependency and interface stability effects produce a different flow pattern from that of the standard system.

When the current emitted by the source has a stable interface, the standard shallow-water (or hydraulic) equations for the thickness $h(\xi, t)$ and depth-averaged $u(\xi, t)$ can be used, where $\xi$ denotes the along-slope coordinate (see Fig. 1). The stable interface is a necessary condition for the propagation of the SW characteristics to the nose. The analysis of the characteristics indicates that at the ridge the flow is "critical" (the Froude number $F=\mathrm{Ri}^{-2}$ of the flow is equal to 1 ) with $u_{i}=\left(g^{\prime} h_{i}\right)^{1 / 2}$, where the subscript $i$ denotes the ridge position. Since $u_{i} h_{i}=q$, we obtain two useful results: (1) from the definition of $B_{0}$, it follows that $u_{i}=B_{0}^{1 / 3}=U$ where $U$ is the characteristic velocity scale; and (2) from the definition of $\mathrm{Ri}$ it follows that $\mathrm{Ri}_{i}=1$, which justifies the assumption of stable interface at the ridge (in agreement with experimental observations). In this case, for a while, the nose propagates in stable conditions and leaves behind a smooth steady-state tail in which $u(\xi)$ increases while $h(\xi)$ and Ri decreases. After the position where Ri is sufficiently small for instabilities to develop, the gravity current is affected by entrainment and the simple SW equations become invalid. Our analysis is focused only on the stable stage, and hence the SW equations are a good approximation.

The propagation of the head with stable initial conditions lacks theoretical understanding and means of prediction.

This paper attempts to close this gap of knowledge concerning the propagation of the nose of the current, $\xi=\xi_{N}(t)$, and corresponding speed $u_{N}(t)$ for a continuously supplied gravity current with a stable interface at the ridge during the "formation" phase by use of the SW framework for gravity currents.

The SW equations are a hyperbolic system, and there is good evidence from solutions of horizontal currents that the nose is a discontinuity where a jump condition can be applied [5]. We implement this idea in the present problem, and show that self-contained results for $u_{N}(t)$ can be obtained. To corroborate the theoretical prediction and sharpen the understanding we compare with laboratory experimental velocity data collected using a particle image velocimetry (PIV) measurement technique.

The paper is organized as follows. In Sec. II we present the SW formulation and derive the theoretical prediction of $u_{N}$. The experimental setup, and methodology for the detection of the propagation of the nose are described in Sec. III. The comparison between theory and measurements is performed in Sec. IV and discussed in Sec. V. Finally, some concluding remarks are given in Sec. VI. All of the symbols that are introduced for this study are listed in Table I to improve the readability of the paper.

\section{SHALLOW WATER MODEL}

We consider a layer of fluid of density $\rho_{c}$ which propagates over a bottom into an ambient fluid of density $\rho_{a}$ driven by the reduced gravity $g^{\prime}$. The flow is two-dimensional. The coordinates $(\xi, \zeta)$ 
TABLE I. Notations.

\begin{tabular}{|c|c|}
\hline Symbol & Definition \\
\hline$a$ & Vertical thickness ratio of the current to the ambient \\
\hline$\alpha$ & Coefficient of proportionality between nose velocity and buoyancy flux \\
\hline$B_{0}$ & Buoyancy flux \\
\hline$c_{ \pm}$ & Characteristics velocity \\
\hline$D$ & Current release distance between the gate and the ridge \\
\hline$F$ & Froude number \\
\hline$g$ & Acceleration of gravity on the Earth's surface \\
\hline$g^{\prime}$ & Reduced gravity of the gravity current \\
\hline$g_{e}^{\prime}$ & Effective reduced gravity of the gravity current \\
\hline$\zeta$ & Normal coordinate to the sloping bottom \\
\hline $\mathcal{H}$ & Height of the gravity current on the vertical coordinate \\
\hline$H$ & Water column thickness \\
\hline$H_{0}$ & Water column thickness upstream of the ridge \\
\hline$h$ & Height of the gravity current on the normal coordinate to the sloping bottom \\
\hline$h_{0}$ & Current thickness at the gate \\
\hline$h_{i}$ & Height of the gravity current at the ridge \\
\hline$h_{N}$ & Height of the gravity current at the nose position \\
\hline$h_{N i}$ & $h_{N}$ at the ridge \\
\hline$\theta$ & Slope angle \\
\hline $\bar{\theta}$ & Mean slope angle from the ridge to the nose position \\
\hline$\theta_{\text {end }}$ & Slope angle at the end of the slope \\
\hline$L$ & Characteristic length scale \\
\hline$M$ & Width of field of view \\
\hline$N$ & Height of field of view \\
\hline$\xi$ & Along-slope coordinate \\
\hline$\xi_{\max }$ & Length of the slope \\
\hline$\xi_{N}$ & Nose position along position \\
\hline$q$ & Flow rate per unit of width \\
\hline$q_{i}$ & Flow rate per unit of width at the ridge \\
\hline$q_{0}$ & Flow rate per unit of width at the gate \\
\hline$R$ & Curvature radius of the concave slope \\
\hline $\mathrm{Ri}$ & Bulk Richardson number \\
\hline $\mathrm{Ri}_{c}$ & Critical bulk Richardson number \\
\hline$\rho_{a}$ & Density of the ambient fluid \\
\hline$\rho_{c}$ & Density of the gravity current \\
\hline$S_{2}$ & Shape factor in Eq. (1) \\
\hline$T$ & Characteristic timescale \\
\hline$t$ & Time \\
\hline $\mathcal{U}$ & Depth-averaged velocity of the gravity current on the horizontal coordinate \\
\hline$U$ & Characteristic velocity scale \\
\hline$u$ & Depth-averaged velocity of the gravity current on the along-slope coordinate \\
\hline$u_{i}$ & Depth-averaged velocity of the gravity current at the ridge \\
\hline$u_{N}$ & Nose velocity \\
\hline$u_{N i}$ & Nose velocity at the ridge \\
\hline$\phi$ & Froude number in the slope coordinate \\
\hline$X$ & Horizontal coordinate \\
\hline$X_{N}$ & Nose horizontal coordinate \\
\hline$X_{i}$ & $X$ at the ridge \\
\hline$Z$ & Vertical coordinate \\
\hline$Z_{i}$ & $Z$ at the ridge \\
\hline$Z_{N}$ & Nose vertical coordinate \\
\hline
\end{tabular}


are along and normal to the sloping bottom, as depicted in Fig. 1. In this system, $u$ is the velocity of the denser layer and $h$ its depth (thickness). The SW model involves the following assumptions: the flow is in the inertial regime (large Reynolds number), and the horizontal length scales are much larger compared to the vertical scales. Vertical accelerations are small so that the pressure is well approximated by the hydrostatic balance. Also, we assume a Boussinesq current and a deep ambient so that the return flow above the current is negligible.

Assuming a stable interface implies negligible entrainment, which justifies the SW approach. The governing equations (details of derivation can be found in [5]) give the volume, continuity, and momentum balance:

$$
\begin{gathered}
\frac{\partial h}{\partial t}+\frac{\partial h u}{\partial \xi}=0 \\
\frac{\partial u}{\partial t}+u \frac{\partial u}{\partial \xi}=g^{\prime}\left(-\frac{\partial h}{\partial \xi} \frac{1}{\cos \theta}+\sin \theta\right) .
\end{gathered}
$$

The system is hyperbolic. The characteristics are

$$
d u \pm\left(\frac{g^{\prime}}{h}\right)^{1 / 2} d h=g^{\prime} \tan \theta d t \quad \text { on } \quad \frac{d \xi}{d t}=c_{ \pm}=u \pm\left(g^{\prime} h\right)^{1 / 2}
$$

The initial condition for the gravity flow is given by a step function of the flux $q$ at $t=0$.

Inspection of the characteristics indicates that at the ridge, denoted by the subscript $i$, the conditions are critical, so that $c_{-}=0$ and $u_{i}=\sqrt{g^{\prime} h_{i}}$.

As for currents over horizontal boundaries, also the gravity currents over sloping boundaries require a jump condition at the nose that can be derived using force balances in a control volume (see $[5,15]$ ) whose boundaries are separated by a small $\delta x=\delta \xi \cos \theta$. By reviewing the analysis, we conclude that for moderate angles $\left(\theta<45^{\circ}\right.$ say) the inclination of the bottom has negligible contribution to the control-volume balances about a thin jump. Therefore, we apply the standard jump conditions on the horizontal and on the slope:

$$
\mathcal{U}=F \sqrt{g^{\prime} \mathcal{H}} \quad \text { and } \quad u=\frac{F}{(\cos \theta)^{3 / 2}} \sqrt{g^{\prime} h} \quad\left[\xi=\xi_{N}(t)\right]
$$

respectively, where $\mathcal{U}$ and $\mathcal{H}$ are respectively the velocity and height of the current on the horizontal $(X, Z) . F=F(a)$ is the Froude number given by an "off-the-shelf" formula [2,16] expressed in terms of the vertical thickness ratio of the current to the ambient $a=h /(H \cos \theta)$, with $H$ the total water column thickness.

Benjamin [16] proposed a relation for $F(a)$ based on analytical considerations:

$$
F(a)=\left[\frac{(2-a)(1-a)}{1+a}\right]^{1 / 2}
$$

and Huppert and Simpson [2] proposed a relation for $F(a)$ that relies on experimental data:

$$
F(a)=\frac{1}{2} a^{-1 / 3} \quad \text { for } a \geqslant 0.075 \text { and } \quad F(a)=1.19 \text { for } a<0.075 .
$$

The motion of the nose of the SW current is governed by two effects: (1) the information at the ridge $\xi=0: u=u_{i}, h=h_{i}$; and (2) the jump condition as given in Eq. (5), namely $u=u_{N}=$ $\phi\left(g^{\prime} h\right)^{1 / 2}$ at $\xi_{N}(t)$, where $\phi=F /(\cos \theta)^{3 / 2}$. The connection between these conditions is provided by a characteristic whose speed is $c_{+}=u+\sqrt{g^{\prime} h}$ and on which $d u=g^{\prime} \tan \theta d t-\sqrt{g^{\prime}} d h / \sqrt{h}$. The trajectories are sketched in Fig. 2. For concave slopes, the exact integration along the characteristic is cumbersome, and we take an averaged angle $\theta$ assuming that the variations along the characteristic are modest. 


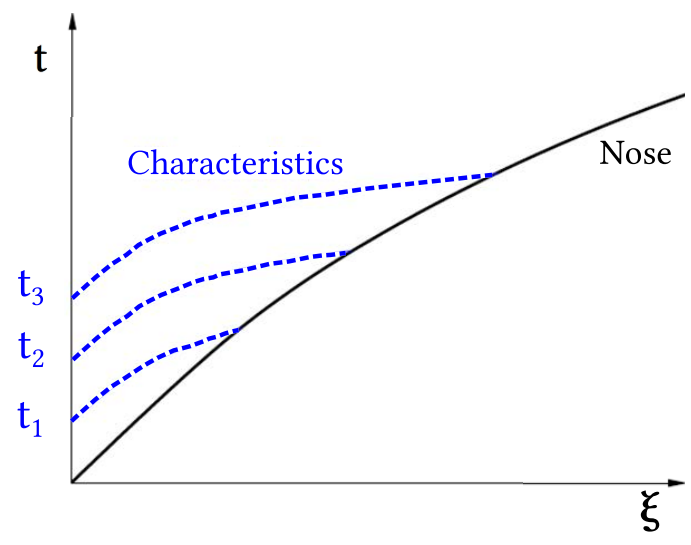

FIG. 2. Sketch of the nose propagation (black line) and of the characteristics released at $t_{1}, t_{2}, t_{3}$ (blue dashed lines).

Supposing that the characteristic is released at the time $t_{k}>0(k \in \mathbb{N})$ and intersects the nose at time $t$, the integral along the characteristic $k$ [cf. Eq. (4)] gives

$$
u_{N}=u_{i}+g^{\prime} \tan \theta\left(t-t_{k}\right)-2 \sqrt{g^{\prime}}\left(\sqrt{h_{N}}-\sqrt{h_{i}}\right) .
$$

We recall that the subscript $i$ denotes the ridge. The intersection of the trajectory of the nose with the characteristic yields

$$
\frac{1}{2}\left(u_{N}+u_{N i}\right) t=\frac{1}{2}\left[u_{i}+u_{N}+\sqrt{g^{\prime}}\left(\sqrt{h_{i}}+\sqrt{h_{N}}\right)\right]\left(t-t_{k}\right),
$$

where $u_{N i}$ is the value of $u_{N}$ at $t=0$ obtained from $q=u_{N i} h_{N i}$. Recalling that $u_{N i}=\phi \sqrt{g^{\prime} h_{N i}}$ and that $u_{i}=\left(g^{\prime} h_{i}\right)^{1 / 2}$ we rewrite the Eqs. (8) and (9) as

$$
\begin{gathered}
u_{N}=\left(1+\frac{2}{\phi}\right)^{-1}\left[3 u_{i}+g^{\prime} \tan \theta\left(t-t_{k}\right)\right], \\
t-t_{k}=\frac{u_{N}+u_{N i}}{2 u_{i}+\left(1+\frac{1}{\phi}\right) u_{N}} .
\end{gathered}
$$

Eliminating $\left(t-t_{k}\right)$ from Eqs.. (10) and (11), we obtain

$$
u_{N}=\left(1+\frac{2}{\phi}\right)^{-1}\left[3 u_{i}+g^{\prime} \frac{u_{N}+u_{N i}}{2 u_{i}+\left(1+\frac{1}{\phi}\right) u_{N}} \tan \bar{\theta} t\right],
$$

where $\bar{\theta}$ is the mean angle from the ridge position to the nose position approximated by $\bar{\theta}=\left(\theta_{0}+\right.$ $\theta) / 2$. The variable $\phi=F / \cos ^{3 / 2} \theta$ is evaluated using both relations (6) and (7). Combining one of these relations for $F(a)$ with the condition of the Froude number, we obtain

$$
u_{N}=\frac{F(a)}{(\cos \theta)^{3 / 2}} \sqrt{g^{\prime} h_{N}}=\phi(a) \sqrt{g^{\prime} h_{N}} .
$$

Equation (12) can be numerically solved starting from the initial values and using a time advancement of $\delta t$, here chosen equal to 0.1 after testing the accuracy with smaller values.

\section{EXPERIMENTAL PROCEDURES}

The experiment, sketched in Fig. 1, and described in detail elsewhere [11], consists of a uniformdensity saline solution injected continuously from an external reservoir on a $25 \mathrm{~cm}$ wide and $20 \mathrm{~cm}$ 
TABLE II. Summary of the experimental parameters of the PIV velocity measurements. The total initial water depth in the channel is $H_{0}=19 \mathrm{~cm}$, the current release distances are $D 1=50 \mathrm{~cm}$ and $D 2=160$ or $200 \mathrm{~cm} . X$ is the horizontal spatial coordinate defined in Fig. 1 and $R$ is the curvature radius also defined in Fig. $1 . \theta_{\text {end }}^{\circ}$ is the slope angle at the end of the slope.

\begin{tabular}{|c|c|c|c|c|c|c|c|c|c|c|}
\hline Expt. & 1 & 2 & 3 & 4 & 5 & 6 & 7 & 8 & 9 & 10 \\
\hline$\theta^{\circ}$ & 15 & 22 & $17.2-X / R$ & $31.5-X / R$ & 15 & 10 & 7 & 15 & 10 & 7 \\
\hline$\theta_{\text {end }}^{\circ}$ & 15 & 22 & 0 & 0 & 5 & 10 & 7 & 15 & 10 & 7 \\
\hline$D(\mathrm{~cm})$ & 160 & & 200 & & & 160 & & & 50 & \\
\hline$q\left(\mathrm{~cm}^{2} \mathrm{~s}^{-1}\right)$ & 32 & & 24 & & & 16 & & & 16 & \\
\hline$g^{\prime}\left(\mathrm{cm}^{2} \mathrm{~s}^{-1}\right)$ & 5.6 & & 4.3 & & & 4.3 & & & 4.3 & \\
\hline$B_{0}\left(\mathrm{~cm}^{3} \mathrm{~s}^{-3}\right)$ & 179 & & 103 & & & 69 & & & 69 & \\
\hline
\end{tabular}

deep channel via a pump through a gate opening of $h_{0}=6 \mathrm{~cm}$. The total length of the horizontal channel is $230 \mathrm{~cm}$ and at its end different inclined boundaries (linear, concave) were employed to enable the flow to accelerate. Further variation parameters were the injected buoyancy flux, the gate position $D$ on the horizontal portion [160 or $200 \mathrm{~cm}(D 2)$ and $50 \mathrm{~cm}(D 1)$ upstream of the ridge] that induce different initial stability conditions (interfacial Richardson numbers) at the ridge. All the parameters of the experiments are reported in Table II. A PIV measurement technique was used to obtain two-dimensional velocity fields in a field of view of $M \times N$, where $M$ and $N$ are respectively 70 and $50 \mathrm{~cm}$ for experiments 2 and 3 and 86 and $65 \mathrm{~cm}$ for the other experiments (cf. Fig. 1). Further details are given in Negretti et al. [11] and Martin et al. [12].

The use of the PIV technique permits to have detailed information about the velocity distribution within the head and give some more options for defining the nose limits and the front speed $[17,18]$. Figure 3(a) gives an instantaneous image of the velocity field for experiment 6 ; the vertical gray line represents the position $\xi(t)$ at which the maximum velocity at the time $t=9 \mathrm{~s}$ is below the value of the reference velocity $U$. The velocity $U$ is hence used as a threshold on the maximum velocity within the current at the time $t$ to detect the nose position [vertical gray line in Fig. 3(b)], which is very close to the position of the nose calculated using the maximum spatial velocity gradient at each time $t$ [red curve in Fig. 3(b)] but exhibiting larger fluctuations. Furthermore, Fig. 3(b) highlights that the maximum of the velocity within the head is upstream of the nose, as also reported by Thomas et al. [17]. Figure 3(c) shows a Hovmöller diagram of the free stream velocity which is a
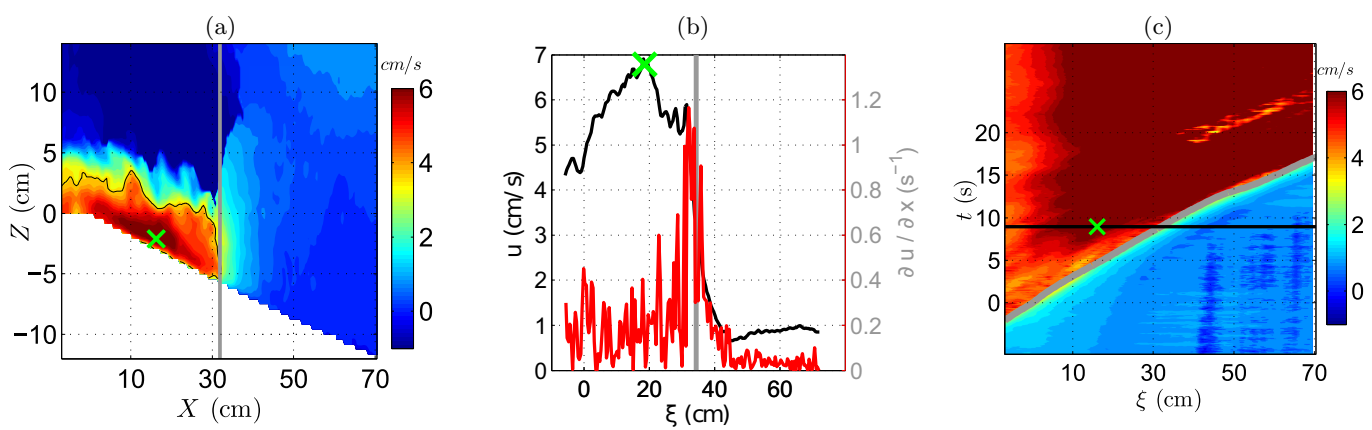

FIG. 3. (a) Instantaneous $\left(t=9 \mathrm{~s}\right.$ ) along-slope velocity for a linear slope of $10^{\circ}$ (experiment 6 ), $u=U$ (black contour). (b) Instantaneous free stream velocity with $\xi$ (black) and spatial derivative of the free stream velocity (red). (c) Hovmöller diagram of the free stream velocity with $\xi$ and $t$, time position of the snapshot (horizontal black line). The nose position is a grey line. The green cross represents the maximum velocity in the head as reported in [11]. 
(a) Exp. 8

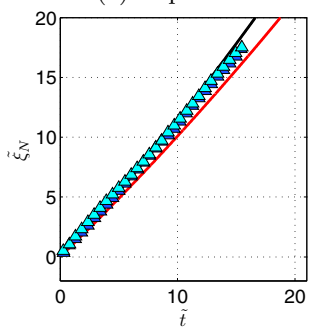

(e) Exp. 8

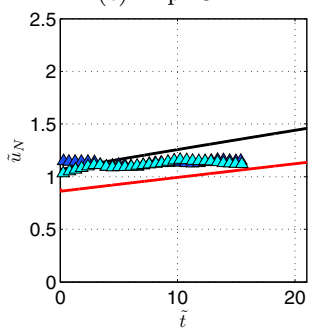

(b) Exp. 9

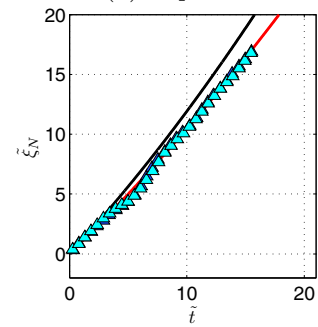

(f) Exp. 9

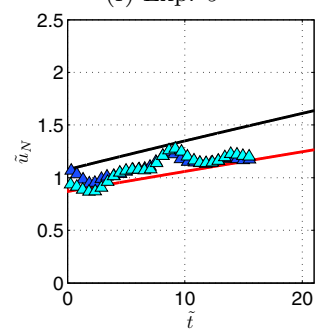

(c) Exp. 10

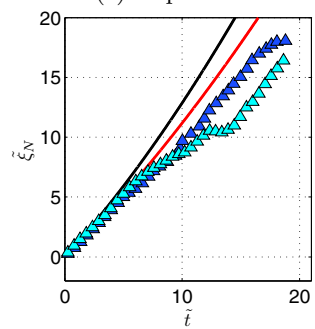

(g) Exp. 10

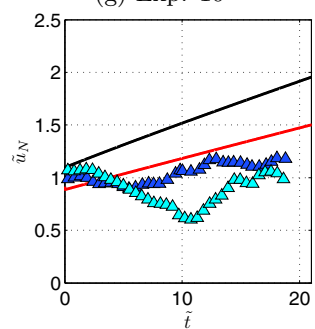

(d) Exp. 5

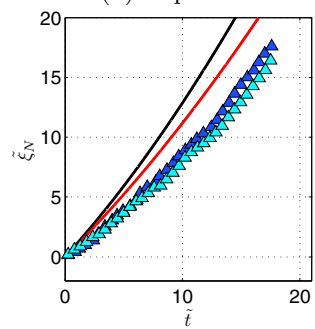

(h) Exp. 5

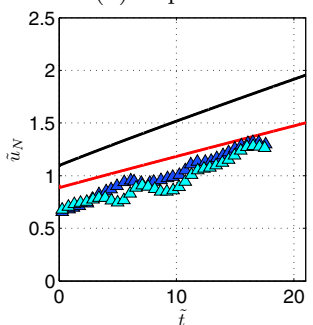

FIG. 4. Nose position (top panels) and nose velocity (bottom panels), for the configurations D1 [(a)-(c) and (e) $-(\mathrm{g})]$ and D2 [(d) and (h)]. Model solution (continuous lines) and experiments (symbols) obtained from a threshold on the free stream velocity (blue triangles) and from the maximum spatial derivative of the free stream velocity (cyan triangles). Theoretical $\tilde{u}_{N}$ and $\tilde{\xi}_{N}$ are computed with $F(a)$, which is estimated with the jump condition (6) (black line) and with Eq. (7) (red line). In (c) the theoretical $\tilde{u}_{N}$ is estimated by using a constant averaged angle $\bar{\theta}$.

function of space and time. The gray line gives the so obtained front advancement $\xi_{N}$ in time $t$. The nose propagation speed is defined as $u_{N}=d \xi_{N} / d t$.

The values of $\xi_{N}$ are binned using a running window of dimensionless time width equal to 1 . The bins are differentiated over a $\Delta \tilde{t}=1$ such that

$$
u_{N}(\tilde{t})=\int_{\tilde{t}}^{\tilde{t}+1} \tilde{\xi}_{N}\left(\tilde{t}^{\prime}\right) d \tilde{t}^{\prime}-\int_{\tilde{t}-1}^{\tilde{t}} \tilde{\xi}_{N}\left(\tilde{t}^{\prime}\right) d \tilde{t}^{\prime},
$$

where variables are made dimensionless using $L=\left(q^{2} / g^{\prime}\right)^{1 / 3}, T=\left(q / g^{2}\right)^{1 / 3}$, and $U=B_{0}^{1 / 3}=$ $\left(q g^{\prime}\right)^{1 / 3}$ and are denoted by . We highly smoothed the nose velocity $u_{N}$ with a running window of $\Delta \tilde{t}=7$.

The rms of the difference between raw velocities and the smoothed velocities is smaller than $0.2 \mathrm{~cm} / \mathrm{s}$.

Negretti et al. [11] defined the head velocity as the maximum PIV velocity within the head at each time $t$ (cf. the cross in Fig. 3) and used it to compare with their free-fall velocity solution. This is highlighted by a cross in Figs. 3(a)-3(c). We point out that this is an alternative definition that is different from the Lagrangian velocity $u_{N}=d \xi_{N} / d t$ (see also Thomas et al. [17]).

\section{COMPARISON WITH EXPERIMENTS}

Figure 4 (top panels) shows the dimensionless nose position $\tilde{\xi}_{N}$ from the ridge against the dimensionless time $\tilde{t}$ for experiments 8,9 , and 10 . We see that the nose position $\tilde{\xi}$ increases practically linearly with time. No significant change is reported when the slope angle changes during the first time period of $5 T$. The slightly different patterns of the nose trajectories observed between the experiments are induced by different initial stability of the tail at the ridge [see $D 1$ configuration in Fig. 4(a) and D2 configuration in Fig. 4(d) for a linear slope of $15^{\circ}$ ]. The nose displacement for 
(a)

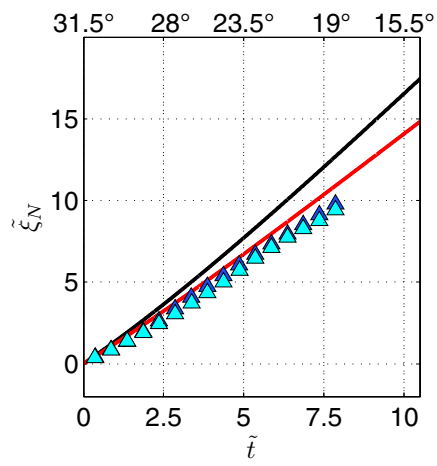

(b)

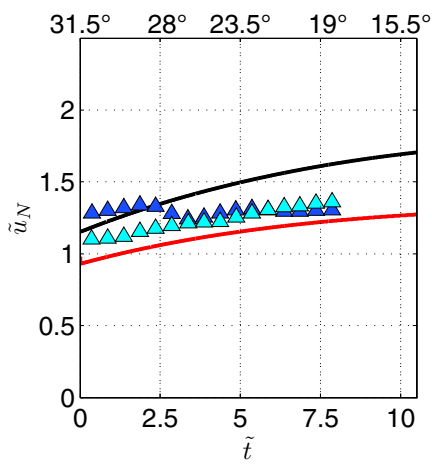

(c)

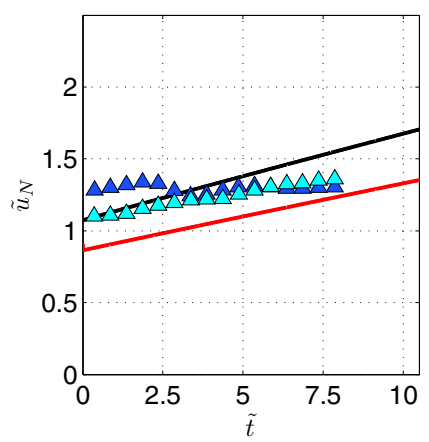

FIG. 5. Nose position (a) and nose velocity [(b) and (c)], for experiment 4 with a concave slope. Symbols are as given in Fig. 4.

the $D 1$ configuration (intermediate initial stable condition) is faster than that in the $D 2$ configuration (initial stable condition). This difference may be ascribed to a higher dilution of the head for the $D 2$ experiment due to the higher release distance.

The dimensionless nose velocity $\tilde{u}_{N}=d \tilde{\xi_{N}} / d \tilde{t}$ is displayed in Fig. 4 (bottom panels). We see that $\tilde{u}_{N}$ increases with $\tilde{t}$, with a rate of less than $20 \%$ for the experiments with $7^{\circ}$ and of $30 \%$ for the experiments on a larger slope angle.

In Fig. 4, for fixed-slope configurations, the theoretical nose propagation (top panels) and speed (bottom panels) are plotted as continuous lines using the two different relations for $F(a)$ : black lines represent Eq. (6) and red lines Eq. (7). The nose displacement and the nose velocity reveal a strong dependency on the Froude condition with an impact of up to $10 \%$ for the displacement and up to $25 \%$ for the velocity.

The theoretical predictions reveal a continuously accelerating current in full accord with the experimental data.

In Fig. 5, for a changing-slope configuration, the theoretical nose propagation (a) and speed (b) are plotted as in Fig. 4. For experiment 4 the travel time of the nose is shorter than for the experiments of Fig. 4 because the field of view of the camera downstream of the ridge is smaller, the characteristic velocity of the nose is higher (due to a higher pump flow rate), and the slope is steeper. The theoretical solution shows a good agreement with the observations. The comparison of Figs. 5(b) and 5(c) shows that both the theoretical prediction for experiment 4 with a changing slope and the prediction with an average constant slope give a correct estimate of the nose displacement and velocity. Nevertheless, the theoretical prediction using a variable angle is much better than the prediction with an average constant slope, particularly for the derivative of the nose velocity. This demonstrates that the theoretical model is able to capture well the effects of the slope.

\section{DISCUSSION}

The presented theoretical SW model of the governing equations of motion combined with a nose-jump (of Benjamin type) condition can predict reasonably well the front displacement and velocity for most of the experiments with both constant and changing slope, paricularly in the first stages, as long as the effects of entrainment and associated drag are negligible. This is a significant improvement of the understanding and of the prediction tools for the motion of a sustained gravity current on a downslope of constant or changing-with-distance slope.

As pointed out in Sec. II, a necessary condition for validity of the SW equations and the corresponding characteristics of the nose is that the tail is not affected by entrainment and drag. 
Consequently, the relevant experiments are those for gravity currents with stable initial conditions at the ridge.

The SW equations (2) and (3) admit a steady-state solution, which corresponds to the tail left behind the nose. Letting $u=u(\xi), h=h(\xi)=q / u(\xi)$, and using the boundary conditions at $\xi_{i}$, we obtain the SW solution

$$
\frac{u}{B_{0}^{1 / 3}}=\left[2 \frac{g^{\prime} \sin \theta}{B_{0}^{2 / 3}} \xi+3-2 \frac{B_{0}^{1 / 3}}{u}\right]^{1 / 2}, \quad h=\frac{q}{u} .
$$

where we set a constant $\theta$ and $\cos \theta \approx 1$ for simplicity, without any loss of generality. The equation for $u$ is implicit, but the behavior is clear, as follows. Defining $\xi=0$ at the ridge we conclude that in the tail $u$ increases monotonically with $\xi$, from the value $B_{0}^{1 / 3}$. Formally, the value of $u$ is bounded only by the length of the slope, $\xi_{\max }$. However, we must consider the stability of the interface of this tail. The local $\operatorname{Ri}(\xi)=g^{\prime} q / u^{3}=B_{0} / u^{3}$ decreases with $\xi$, and hence the interface is expected to become unstable at some $\xi_{c}$ where $\mathrm{Ri}=\mathrm{Ri}_{c}<0.25$. Using (15) we obtain the estimate for the length of the stable tail:

$$
\xi_{c} \approx\left(\frac{1}{\mathrm{Ri}_{c}^{2}}-3\right) \frac{B_{0}^{2 / 3}}{2 g^{\prime} \sin \theta} \approx \frac{5}{\sin \theta}\left(\frac{q^{2}}{g^{\prime}}\right)^{1 / 3} .
$$

We conclude that the stability criterion limits the length of the SW smooth tail, and also the maximum value of $u$, to $\left(B_{0} / \mathrm{Ri}_{c}\right)^{1 / 3}$. With a safety margin, based on the results of Negretti et al. [11], we infer that the speed of the current in the stable tail is in the range $[1,2] B_{0}^{1 / 3}$ and, since the instability produces entrainment and drag, we argue that the speed of the current in the unstable $\mathrm{Ri}<\mathrm{Ri}_{c}$ domain will not exceed $2 B_{0}^{1 / 3}$. From Eq. (16) the typical scaled $\tilde{\xi}$ and $\tilde{t}$ of the propagation in the stable domain are expected to be of the order of $5 / \sin \theta$. For a slope of $15^{\circ}, 5 / \sin \theta=19$.

The experiments of Negretti et al. [11] confirm these predictions: observed currents starting with $\mathrm{Ri} \approx 1$ at $\xi_{i}$ have a stable interface over a significant distance, but develop suddenly KelvinHelmholtz $(\mathrm{KH})$ vortices for $\xi>\xi_{c}$. At $\xi_{c}$ the experimental values of entrainment and bottom drag increase with $\xi$. The density of the current is diluted, and the effective reduced gravity $g_{e}^{\prime}$ is smaller than the reduced gravity at the gate, $g_{0}^{\prime}$. They are related by the conservation of the buoyancy flux, $g_{e}^{\prime} q_{i}=g_{0}^{\prime} q_{0}=B_{0}$, where $q_{i}$ is the flow rate per unit of width at the ridge, $q_{0}$ at the gate. If no mixing occurred between the gate and the ridge, then $q_{i}=q$ and $g^{\prime}=g_{e}^{\prime}$, which is what it has been assumed within this paper. The behavior of the flow is very different from the simple SW results (which assume $E=c_{D}=0$ ); in particular, $u$ decreases and $h$ increases with $\xi-\xi_{c}$. Some distance after $\xi_{c}$, the observed Kelvin-Helmholtz billows are so large that a depth-averaged approach becomes inappropriate.

A further case, as mentioned in the Introduction, is that of a current with unstable conditions at the ridge, as considered for example in Britter and Linden [8], Ellison and Turner [9], and Pawlak and Armi [10]. Britter and Linden [8] reported that, given a buoyancy flux $B_{0}$, after an initial short acceleration phase the forces are quickly balanced (within $10 h_{i}$ ) and the front enters an established regime where the velocity $u_{N}$ is constant, scaling as $u_{N}=\alpha\left(B_{0}\right)^{1 / 3}$, with $\alpha=1.5$ and eventually with $\alpha$ a function of the Froude number at the nose and of the slope angle. Tokyay and García [19] obtain $\alpha=1.11-1.17$ in their numerical simulations with slope angles $\theta<3^{\circ}$. In our experiments we report $\alpha=1.2-1.3$ for $\tilde{\xi}>10$. This difference may be attributed to the depth ratio, which is larger in the experiments of Britter and Linden, resulting in a larger Froude number at the nose as compared to our experiments.

\section{CONCLUDING REMARKS}

We presented a theoretical model which explains and predicts the motion of the nose of a downslope gravity current sustained by a source, and compared the model with experiments. One important conclusion is that the propagation of the nose is strongly affected by the Richardson 
number at the ridge and along the slope. We showed that for certain initial conditions the stability of the interface can be maintained for a significant distance of propagation.

We demonstrate that, in the case of a stable interface at the ridge, $u_{N}$ is a match between a jump condition at the moving nose and information carried by the characteristic from the ridge through the stable tail. This $u_{N}$ increases with time (and downslope distance), Ri decreases with time, and hence at some position the stable $u_{N}$ switches to the entrainment-drag dominated value $1.5 B_{0}^{1 / 3}$. It is difficult to assess the accuracy of the theoretical prediction. First, there is some uncertainty concerning the Froude jump condition $F=F(a)$. The semiempirical Huppert and Simpson [2] formula gives better agreement than the more rigorous Benjamin formula; this, however, is consistent with the behavior of gravity currents on a horizontal bottom. Second, and more important, are the limitations of the experimental data. The position $\xi_{N}$ of the nose is not a sharp experimental variable, and the calculation of the time derivative $u_{N}$ yields noisy data that need strong smoothing. We employed several methods of measurement and smoothing, but some discrepancies remain, which could be improved for example using high-resolution Navier-Stokes simulations.

The Lagrangian velocity of propagation of the nose, $u_{N}=d \xi / d t$, is significantly smaller than the interior maximal velocity in the head. This difference may be important in geophysical and environmental applications, where both position of the current and internal convection are of interest. The details of the transition from the SW regime to the entrainment-drag regime require a complex investigation, which is left for future work.

\section{ACKNOWLEDGMENTS}

This work has been partially supported by the LabEx Tec 21 (Investissements d'Avenir, Grant Agreement No. ANR-11-LABX-0030). We are grateful to P. Jarrin, S. Mercier, and S. Viboud for their technical support for the experiments. Special thanks go to E. J. Hopfinger for useful discussions.

[1] E. J. Hopfinger, Snow avalanche motion and related phenomena, Annu. Rev. Fluid Mech. 15, 47 (1983).

[2] H. E. Huppert and J. E. Simpson, The slumping of gravity currents, J. Fluid Mech. 99, 785 (1980).

[3] T. J. Sherwin and W. R. Turrell, Mixing and advection of a cold water cascade over the Wyville Thomson ridge, Deep Sea Res. 52, 1392 (2005).

[4] J. W. Rottman and J. E. Simpson, Gravity currents produced by instantaneous releases of a heavy fluid in a rectangular channel, J. Fluid Mech. 135, 95 (1983).

[5] M Ungarish, An Introduction to Gravity Currents and Intrusions (CRC, Boca Raton, 2009).

[6] C. G. Johnson and A. J. Hogg, Entraining gravity currents, J. Fluid Mech. 731, 477 (2013).

[7] J. B. Klemp, R. Rotunno, and W. C. Skamarock, On the dynamics of gravity currents in a channel, J. Fluid Mech. 269, 169 (1994).

[8] R. E. Britter and P. F. Linden, The motion of the front of a gravity current travelling down an incline, J. Fluid Mech. 99, 531 (1980).

[9] T. H. Ellison and J. S. Turner, Turbulent entrainment in stratified flows, J. Fluid Mech. 6, 423 (1959).

[10] G. Pawlak and L. Armi, Mixing and entrainment in developing stratified currents, J. Fluid Mech. 424, 45 (2000).

[11] M. E. Negretti, J.-B. Flòr, and E. J. Hopfinger, Development of gravity currents on rapidly changing slopes, J. Fluid Mech. 833, 70 (2017).

[12] A. Martin, M. E. Negretti, and E. J. Hopfinger, Development of gravity currents on slopes under different interfacial instability conditions, J. Fluid Mech. 880, 180 (2019).

[13] J. S. Turner, in Buoyancy Effects in Fluids (Cambridge University Press, Cambridge, 1973), p. 405.

[14] J. S. Turner, Turbulent entrainment: The development of the entrainment assumption, and its application to geophysical flows, J. Fluid Mech. 173, 431 (1986). 
[15] M. Ungarish and A. J. Hogg, Models of internal jumps and the fronts of gravity currents: Unifying twolayer theories and deriving new results, J. Fluid Mech. 846, 654 (2018).

[16] T. B. Benjamin, Gravity currents and related phenomena, J. Fluid Mech. 31, 209 (1968).

[17] L. P. Thomas, S. B. Dalziel, and B. M. Marino, The structure of the head of an inertial gravity current determined by particle-tracking velocimetry, Exp. Fluids 34, 708 (2003).

[18] H. I. S. Nogueira, C. Adduce, E. Alves, and M. J. Franca, Dynamics of the head of gravity currents, Env. Fluid Mech. 14, 519 (2014).

[19] T. E. Tokyay and M. H. García, Effect of initial excess density and discharge on constant flux gravity currents propagating on a slope, Env. Fluid Mech. 14, 409 (2014). 